PREPRINT - R. Vidoni, R. Gallo, G. Ristorto, G. Carabin, F. Mazzetto, L. Scalera, A. Gasparetto, ByeLab : an Agricultural Mobile Robot Prototype for Proximal Sensing and Precision

Farming, Proceedings of the ASME 2017 International Mechanical Engineering Congress and Exposition, IMECE2017, November 3-9, 2017, Tampa, Florida, USA

\title{
BYELAB : AN AGRICULTURAL MOBILE ROBOT PROTOTYPE FOR PROXIMAL SENSING AND PRECISION FARMING
}

\author{
Renato Vidoni* \\ Raimondo Gallo \\ Gianluca Ristorto \\ Giovanni Carabin \\ Fabrizio Mazzetto
}

\author{
Faculty of Science and Technology \\ Free University of Bozen-Bolzano \\ Bolzano, 39100, Italy \\ Email: renato.vidoni@unibz.it
}

\author{
Lorenzo Scalera \\ Alessandro Gasparetto \\ DPIA - University of Udine \\ Udine, 33100, Italy
}

\section{ABSTRACT}

At today, available mechatronics technology allows exploiting smart and precise sensors as well as embedded and effective mechatronic systems for developing (semi-)autonomous robotic platforms able to both navigate in different outdoor environments and implementing Precision Farming techniques. In this work, the experimental outdoor assessment of the performance of a mobile robotic lab, the ByeLab - Bionic eYe Laboratory - is presented and discussed. The ByeLab, developed at the Faculty of Science and Technology of the Free University of Bolzano (I), has been conceived with the aim of creating a (semi-)autonomous robotic system able to sense and monitor the health status of orchards and vineyards. For assessing and measuring the shape and the volume of the canopy, LIDAR technology coupled with ad-hoc developed algorithms have been exploited. To validate the ByeLab different experimental tests have been carried out. In addition to the in-lab and structured environments experimental tests that allowed to tune the algorithms, in this work the assessment of its capabilities in particular the sensoric system has been made outdoor controlled environment tests.

\footnotetext{
*Address all correspondence to this author.
}

\section{INTRODUCTION \\ 1 Introduction}

Precision Agriculture (PA) is one of the most important managing strategies in crop production. Its principles can be applied on crops, vegetative stage and agricultural operation and activity, from the tillage to the application of fertilizers [1], from the soil characterization to the crop monitoring. PA activities can be grouped into four sequential phases involving several technologies, ranging from Electronics and Informatics to Mechanics, to: (1) collect field and environmental data, (2) elaborate data and integrate them into an information system (automatic operational monitoring system) tailored to better adapt to the farm requirements $[2,3]$, (3) take decision on the crop management and then (4) drive the machines and/or adapt the action of the actuators in all the agricultural operations. Indeed, thanks to a continuous adjustment of the settings of the implements used for a crop (principle of "variable-rate application"), PA gives many opportunities for increasing the field yields and decreasing the production costs, or, in other words, to make agriculture more efficient but sustainable [4].

The practice of precision agriculture has been possible by introducing new technologies such as Global Navigation Satellite Systems (GNSSs). These systems allow geo-referencing data 
(phase 1) and machines (phase 4) that use the information derived from the interpretation of the collected data. As evidenced above, the acquisition of field and environmental data, possibly geo-localized, is the first step for implementing PA within a farm-company and, together with the management of all these data, belongs to the so-called crop-, operative-, environmentaland performance-monitoring.

This study focuses the attention just on this step and presents a crop-monitoring mobile laboratory making a combined use of two different types of optical sensors useful to collect automatically field-data and obtain many important agronomic information.

The remainder of this paper is as follows: the mobile platform is firtly described together with the developed hardware and software; then, the algorithm work-flow implemented to manage the sensors data is illustrated. After that, the following sections deal with the experimental tests performed to validate the system with a simplified representation of an orchard row. Conclusions and future work are finally highlighted.

\section{THE BYELAB - Bionic eYe Laboratory - MOBILE PLAT- FORM}

A mobile vehicle to be used for plants' sensing has to satisfy some basic technical requirements: (a) being able to move nimbly within the orchards, travelling off-road and perform the turning manoeuvres even in steep hill-sides; (b) being easily transportable inside the investigation site; (c) allowing to lodge on it the individuated crop monitoring sensors by means of an adhoc structure. Moreover, the robotic system has to grant a high level of reliability and safety for eventual humans near it and for the environment in general. The safety toward humans and natural habitat becomes more and more important when considering a fully-autonomous system where no human intervention is required. Keeping in mind these requirements, a tracked binscarrier (the NEO Alpin by Windegger S.r.l., Lana, Bolzano, Italy) has been used as mobile base for this robot, after having been suitably adapted for this purpose. The choice went toward this solution because this system is electrically-driven and wirelessly remote-controlled, it is compact (length $\mathrm{x}$ width: $1.14 \mathrm{x} 1.12 \mathrm{~m}$ ) and allows a high carrying capacity $(500 \mathrm{~kg})$ with respect to its mass $(250 \mathrm{~kg})$, see Fig. 1(a). Moreover, the tracked configuration grants the widest versatility of use for this robot, thanks to the great contact area with the ground which results in a low pressure on the ground and a high grip. Another interesting characteristic of the chosen bins-carrier is its low overall height and the consequent low height of its center of mass from the soil, due to the original scope of this machine, i.e. leaving the capability to self-load and transport bins for fruit-collection. Since the orchard monitoring is the target application and its map and tree position layout are well known so that they can be considered a semi-structured environment, no navigation problems are

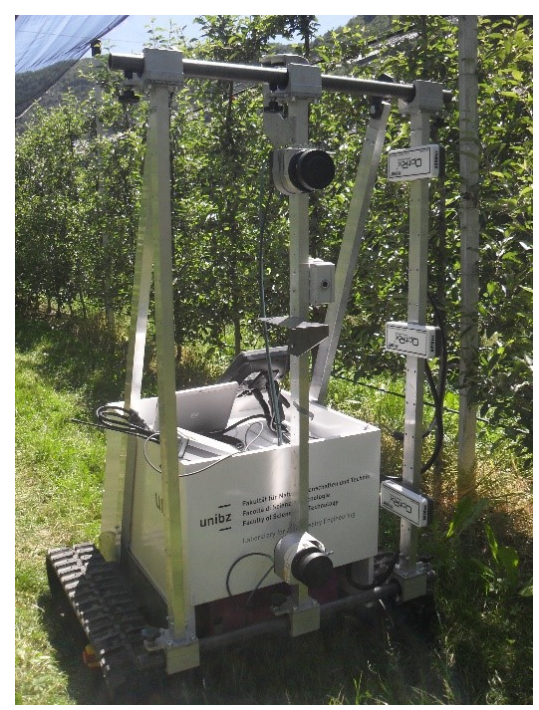

(a)

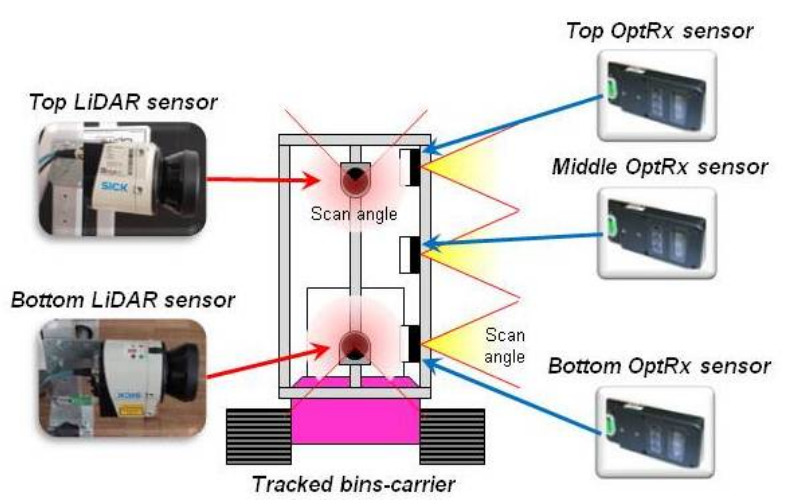

(b)

FIGURE 1: ByeLab mobile robot equipped with sensors. a) lateral view; b) frontal view and sensors placement (NB: for a better clearness, only a row of OptRX sensors is represented).

present. Thus, there is no necessity of special hardware and software architectures for controlling the mobile robotic platform. Then, in this work, more attention has been paid on the study, development and implementation of the mobile robotic lab proprioand extero- ceptive sensing capabilities for a proper assessment of both geometric and health parameters of crops.

For housing and fixing the different sensors, a vertical adjustable frame made of steel joints and aluminium tubular beams has been designed and installed on it, (Fig. 1); in such a manner, given an orchard to be evaluated with its height and inter-row distance, the system can be adapted by choosing a proper configuration of the beams and the best height for the sensors. To properly reconstruct the vegetative state of the plant (i.e. canopy area and volume, and vegetative index), data from different sensors have 
been combined together. More in details, two SICK LMS111 LiDAR sensors are used to detect the surface profile of the plants. They are vertically aligned and mounted at two different heights, on the adjustable vertical frame, and oriented as shown in Fig. 1(b), so the scan angles are directed to both the sides (i.e. the sky and the ground are not perceived). The LMS111 LiDARs fall within the short-range devices and have a maximum scan radius of 20 meters and a working-angle of 270 . They are active sensors, thus allowing to avoid possible problems related to different environmental conditions, e.g. sunlight, clear sky. The crops Vegetative Index (VI), a parameter that gives information about the crop health, is obtained from the six AgLeader OptRx ACS430 crop sensors. They are mounted on the left and right side of the mobile robot (three sensors per side) at three different heights (see Fig. 1(b)). Every sensor is able to record and measure real-time information such as the Vegetative Indexes NDVI (Normalized Difference Vegetation Index) and NDRE (Normalized Difference Red Edge Index), a measurement range of 0.25$2.00 \mathrm{~m}$ and a frequency of $5 \mathrm{~Hz}$. In addition, in order to track the robot speed and position both a sonar sensor and a RTK GNSS have been installed. The former allows to have the position data of the ByeLab in terms of distance from a movable target (e.g. a vertical panel) inner a building or lab, where the GNSS could not work properly; the latter provides GNSS position with a 1 $\mathrm{cm}$ accuracy thanks to a separate fixed base-station. Finally, with the aim of having information also on the mobile robot orientation on uneven terrains, it has been equipped with a LMRK 10 AHRS Inertial Measurement Unit by Gladiator Technologies (http://www.gladiatortechnologies.com/). It directly provides the robot orientation expressed in terms of roll, yaw and pitch angles and, furthermore, gives data from its gyroscope, accelerometers and magnetometer. In Fig. 2 the con-

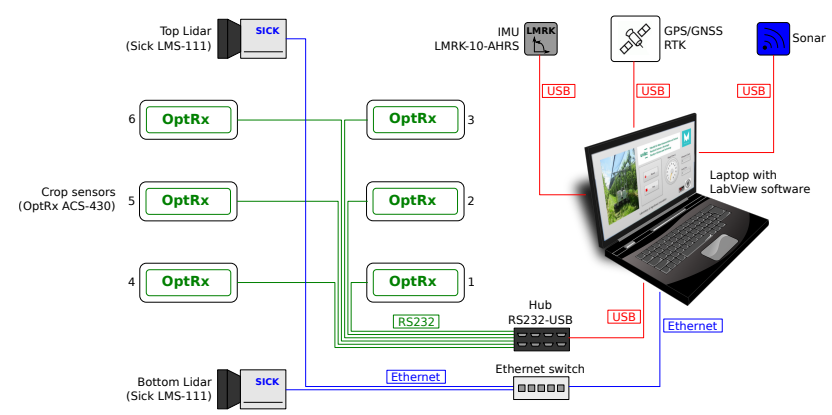

FIGURE 2: Scheme of the connections of the different sensors to the main PC unit.

nection diagram of the data acquisition and control system is reported. The core is a standard laptop, on which the acquisition software program, developed in a LabView ${ }^{\mathrm{TM}}$ environment, runs. The choice to use a laptop, easily installable and removable from the robot as well as easily replaceable, has been made to keep the whole system and equipment as simple as possible, allowing also to non-skilled operators to be able to work with the ByeLab and its software. As a consequence, a mechatronic system that integrates and controls the different equipment has been designed to interface the sensors using different buses on a common central platform. In particular, the two LiDAR sensors are connected together through a Ethernet-switch and this to the laptop Ethernet port. The crop sensors, which communicate via a serial bus, are connected to a RS232-USB hub and then to the laptop by a USB port. Similar solutions have been chosen for the other sensors, i.e. IMU, sonar and RTK GPS. All the crop sensors are powered directly by the main $12 \mathrm{~V}$ battery of the ByeLab, while the proprioceptive sensors, i.e. the IMU, GPS and sonar, are powered directly by the $5 \mathrm{~V}$ made available by the USB laptop ports of the laptop.

\subsection{Acquisition and Post-processing Software}

To acquire data from the different sensors and properly record and synchronize them for the subsequent post-processing phase, a real-time Labview ${ }^{\mathrm{TM}}$ application program has been developed. As data are sent through an Ethernet connection from the LiDAR sensors and through a Serial communication for the others, different transmission speeds had to be managed. Then, a sampling rate of $50 \mathrm{~Hz}$ for the two LiDARs and a lower acquisition frequency for the others have been considered.

The data processing algorithm has been developed in Matlab environment, Fig. 3.

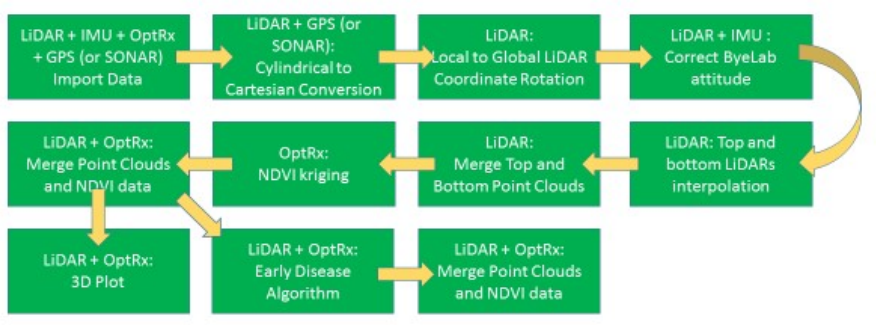

FIGURE 3: Workflow of the developed algorithm.

The algorithm firstly converts the point cloud coordinates obtained by means of LiDAR scans (i.e. the points are individuated by the scan angle $\alpha$ and the distance of the target point from 
the sensor $\rho$ ) from a cylindrical reference system to a Cartesian one as follows:

$$
\left\{\begin{array}{l}
x=\rho \cos \alpha \\
y=\rho \sin \alpha \\
z=\sum_{i} v_{i} \Delta t
\end{array}\right.
$$

where $v$ is the robot speed and $\Delta t$ the time interval for considered acquisition. The coordinates, which are referred to the sensor reference system, are then translated to the robot reference system by taking into account the LiDAR sensors mounting position on the robot. Finally, possible deviations of the robot from the trajectory path and oscillations due to the terrain conformation are corrected by considering the IMU information.

At this point, points at different distance from the sensors generate a non-uniformly distributed $y-z$ grid. Each LiDAR data is interpolated on a uniformly distributed $y-z$ grid, excluding useless regions, by means of a merging algorithm. In this way, a 3D-surface map is obtained as follows:

$$
\begin{cases}x_{i}=\frac{x_{\text {high }, i}+x_{\text {low }, i}}{2} & , \text { for }\left|x_{\text {high }, i}-x_{\text {low }, i}\right| \leq \text { tol } \\ x_{i}=x_{\text {high }, i} & , \text { for }\left|x_{\text {high }, i}-x_{\text {low }, i}\right|>\text { tol } \wedge x_{\text {high }, i}<x_{\text {low }, i} \\ x_{i}=x_{\text {low }, i} & , \text { for }\left|x_{\text {high }, i}-x_{\text {low }, i}\right|>\text { tol } \wedge x_{\text {high }, i}>x_{\text {low }, i} \\ y_{i}=y_{\text {high }, i}=y_{\text {low }, i} & \\ z_{i}=z_{\text {high }, i}=z_{\text {low }, i} & \end{cases}
$$

where $t o l$ is the tolerance parameter expressed in $m m$, and sub-

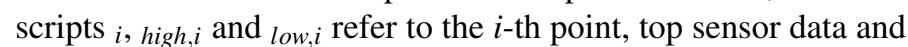
bottom sensor low, respectively. Finally, a 3D mesh surface is obtained by interpolating the point cloud, and the objects area and volume (the half of the object) can be computed by using previously developed algorithms.

The plants' health estimation is obtained by means of the OptRX sensors. By processing the acquired normalize difference vegetation index (NDVI), a normalized ratio of the NIR (near infra-red) and red bands [5], it is possible to understand the differences in vegetative development among plant groups (e.g. high NDVI values are recognized as dense vegetation). The NDVI maps, that show differences in vegetative development among plant group, can be merged and superimposed with the previous volume estimations and maps elaborations (note that in the interpolation of the NDVI map, the same grid used for the LiDAR $y-z$ interpolation is adopted), Fig. 6(b).

Moreover, by correlating the vegetation thickness (e.g. dividing it in ranges) with the NDVI index, it is possible to create additional new simplified maps that allow to better highlight different healthy and unhealthy regions of the vegetation. To improve the visual illustration of the vigour map, a new diagnostic algorithm has been developed and implemented. The algorithm combines the NDVI index and the vegetation thickness as illus-

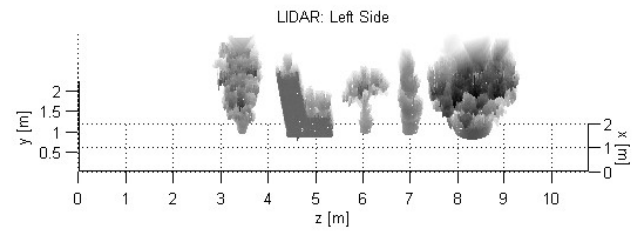

(a)

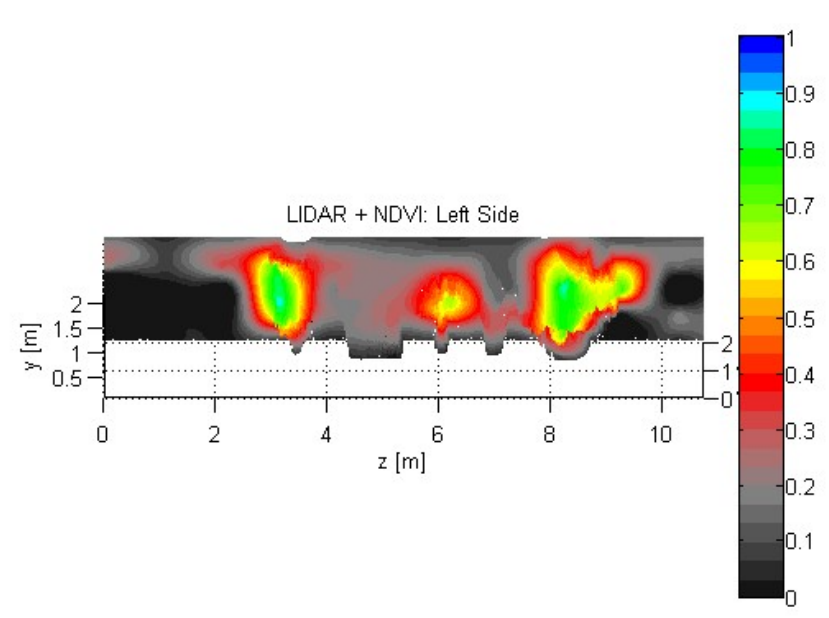

(b)

FIGURE 4: Orchard row 3D representations: a) Lateral-LinearStereoscopic LiDAR vision; b) LiDAR and OptRx merged data.

trated in the matrix of Fig. 5(a). The matrix columns discriminate the NDVI range in three different fields:

$$
\begin{aligned}
& \text { NDVI }<0.6 \\
& 0.6 \leq \mathrm{NDVI}<0.8 \\
& \text { NDVI } \geq 0.8
\end{aligned}
$$

while the matrix rows discriminate the vegetation thickness in three different ranges:

$$
\begin{aligned}
& \mathrm{t}<0.1 \mathrm{~m} \\
& 0.1 \leq \mathrm{t}<0.2 \mathrm{~m} \\
& \mathrm{t} \geq 0.2 \mathrm{~m}
\end{aligned}
$$

Therefore, the green cells of the matrix correspond to the healthful vegetation; the yellow areas characterize an early situation of stress that requires further investigation; the red area coincides 
with a very unhealthy vegetation. Finally, the black cell encompasses the area without foliage. An example is given in Fig.5(b).

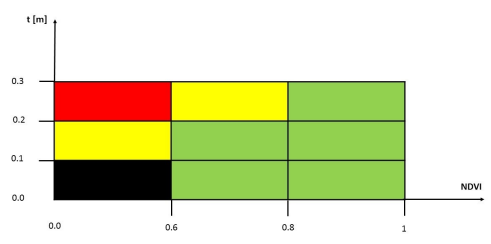

(a)

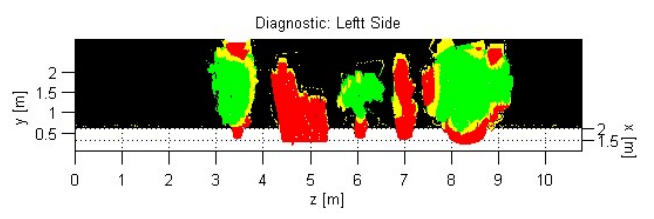

(b)

FIGURE 5: Orchard row 3D representations: a) Early disease diagnostic algorithm matrix; b) Diagnostic algorithm representation.

\section{EXPERIMENTAL TESTS}

\subsection{Outdoor controlled scenario}

The ByeLab has been firstly tested in an outdoor controlled scenario, see Fig. 6, to check and tune the hardware, the acquisition system and the implemented processing algorithms with a focus on the vigour maps and on the early diagnostic algorithm; moreover, new methodologies to represent the volume and health status of an entire crop field are proposed.

In this test an orchard row is simulated, aligning on the same line four plants and a wood structure. The ByeLab travels at a constant speed $(0.5 \mathrm{~m} / \mathrm{s})$ on both side of the row, recording the data measured by the sensors. A Faro Focus 3D X330 HDR Terrestrial Laser Scanner (TLS, $2 \mathrm{~mm}$ distance accuracy) has been exploited to compare the data about the canopy thickness. Four scanning positions have been considered to obtain the 3D reconstruction with the TLS.

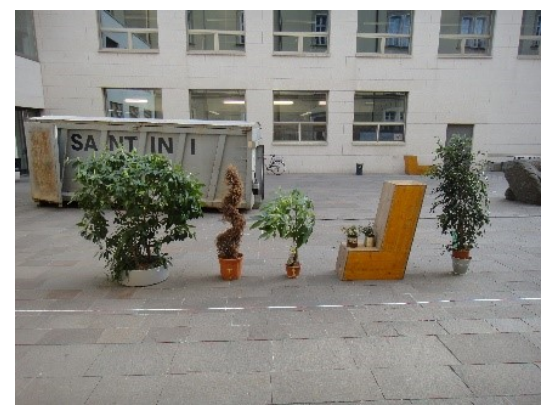

(a)

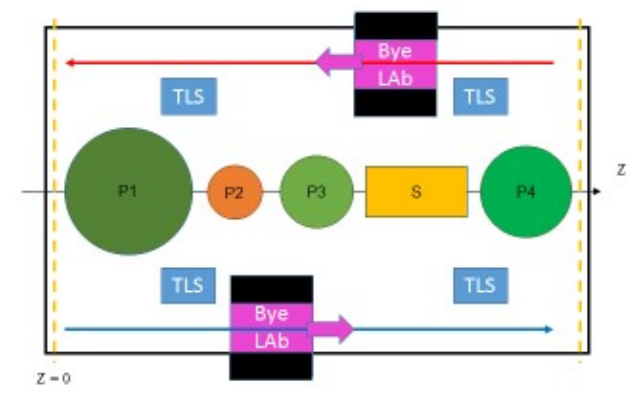

(b)

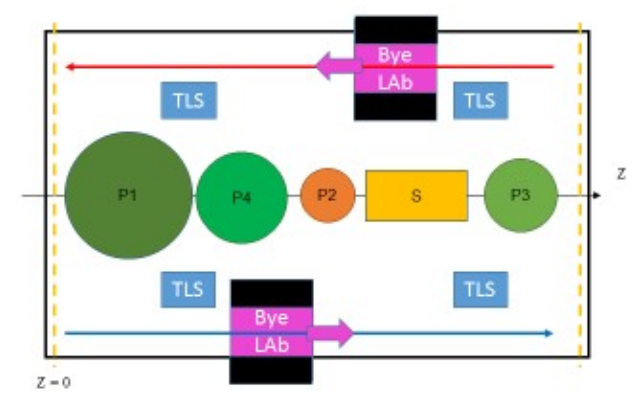

(c)

FIGURE 6: Experimental layout; (b) first layout; (c) second layout. 


\subsection{Validation Results}

The canopy thickness evaluated by the TLS and the ByeLab, for both the experimental layouts, Fig. 6 .

The correlation between the TLS and the ByeLab canopy thickness measurements is presented in Fig. 7 and 8 and the computed values are $\mathrm{R} 2=0.83$ and $\mathrm{R} 2=0.89$ respectively for the first and the second layout.

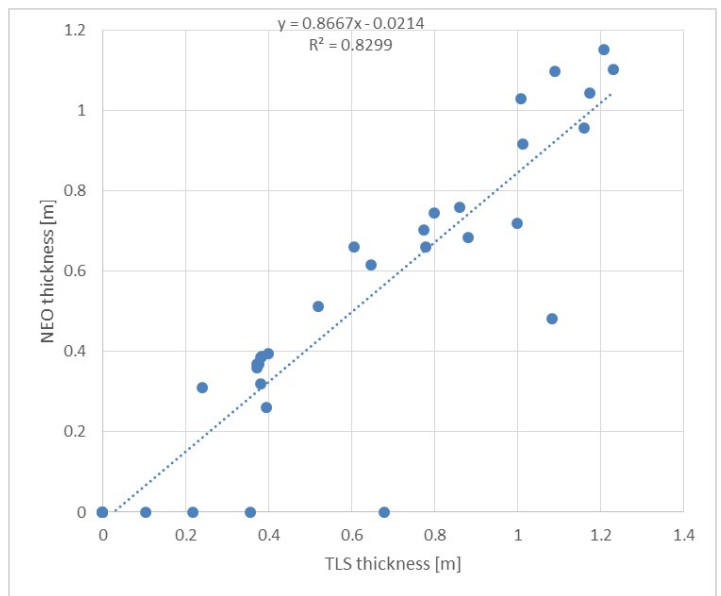

(a)

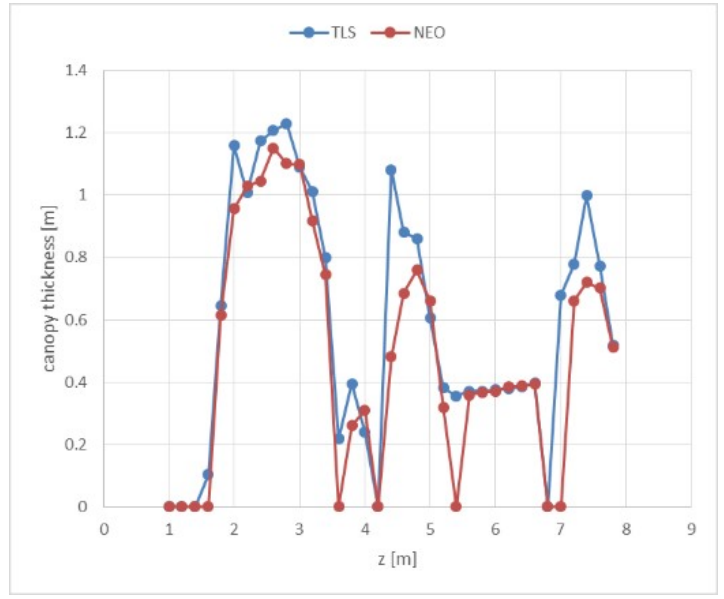

(b)

FIGURE 7: Canopy thickness evaluated by the TLS and the ByeLab for the first experimental layout: a) Correlation between the measurements; b) Trend along the orchard row.

Fig. 7(b) and 8(b) illustrate the measured canopy thickness along the simulated orchard row. The solid structure is correctly measured, but discrepancies in plant measurement occur. Indeed, ByeLab seems to underestimate the plants' thickness, probably due to the windy conditions experienced during the tests.

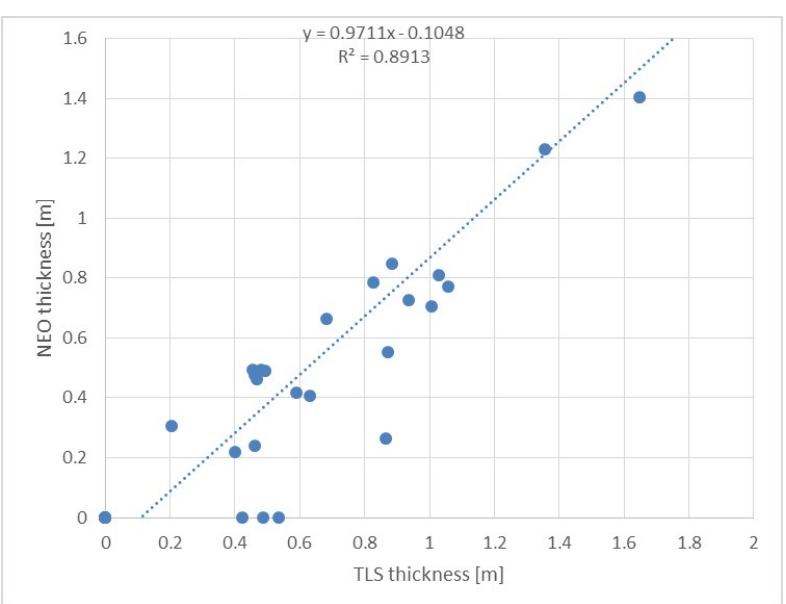

(a)

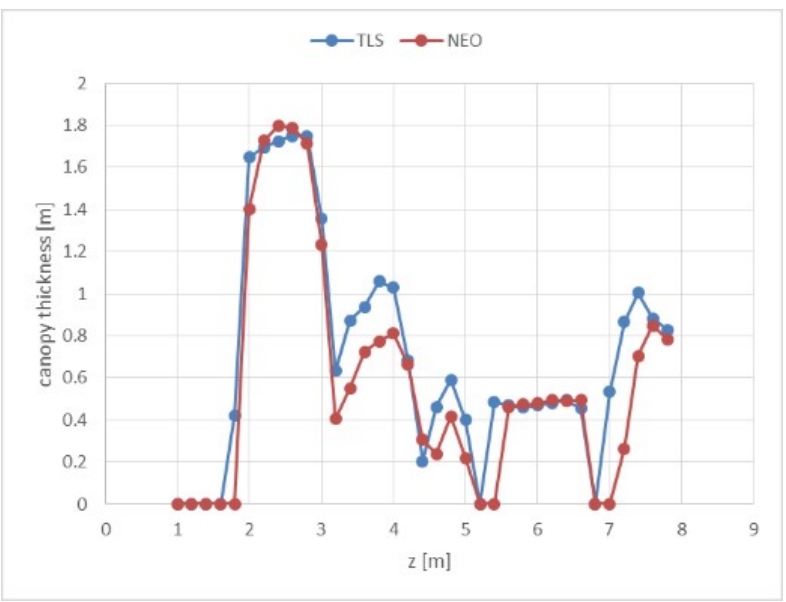

(b)

FIGURE 8: Canopy thickness evaluated by the TLS and the ByeLab for the second experimental layout: a) Correlation between the measurements; b) Trend along the orchard row.

\section{CONCLUSIONS AND FUTURE WORK}

This work presents the design, development and implementation of a (semi-)autonomous mobile robotic platform equipped with state-of-the-art sensors for the volume, vegetation and health estimation of plants inner an orchard: the ByeLab. After the description of the mobile platform and of the hardware architecture, the implemented vision system and used sensors chosen and installed on the ByeLab have been presented together with the procedure and developed algorithms for data acquisition and processing. The system has been tested in an outdoor semi-structured environment showing interesting results and performance, which allowed us to go on with outdoor intensive tests and robotic application. Currently, the robustness of the measurements to terrain and atmosphere non-ideal conditions are 
evaluated. Moreover, another point of study is the management of the large amount of data acquired to create the thematic maps useful for an early disease detection of the plants of an orchard. Future work will be devoted to the extension of the mobile ByeLab robot capabilities in terms of navigation and obstacle avoidance (exploitation of ROS), with the aim of applying it in other less structured environments.

\section{ACKNOWLEDGMENT}

The research leading to these results has been supported by the Monalisa project, which was funded by the Autonomous Province of Bozen-Bolzano.

\section{REFERENCES}

[1] Pahlmann, I., Bttcher, U., and Kage, H., 2016. "Evaluation of small site-specific $\mathrm{n}$ fertilization trials using uniformly shaped response curves". European Journal of Agronomy, 76, pp. 87-94.

[2] Calcante, A., and Mazzetto, F., 2014. "Design, development and evaluation of a wireless system for the automatic identification of implements". Computers and Electronics in Agriculture, 101, pp. $118-127$.

[3] Kruize, J., Wolfert, J., Scholten, H., Verdouw, C., Kassahun, A., and Beulens, A., 2016. "A reference architecture for farm software ecosystems". Computers and Electronics in Agriculture, 125, pp. 12-28.

[4] Gavioli, A., de Souza, E., Bazzi, C., Guedes, L., and Schenatto, K., 2016. "Optimization of management zone delineation by using spatial principal components". Computers and Electronics in Agriculture, 127, pp. 302-310.

[5] Povh, F., and de Paula, W. dos Anjos, G., 2014. "Optical sensors applied in agricultural crops". Optical Sensors - New Developments and Practical Applications. 